\title{
Development of Laser Ablation with Filter (LAF) sampling method for micro-scale isotopic analysis of geologic materials
}

\author{
YUKI MASUDA $^{1 *}$, TETSUYA YOKOYAMA ${ }^{1}$, SATOKI
} OKABAYASHI $^{2}$, AKIRA ISHIKAWA ${ }^{1}$

${ }^{1}$ Tokyo institute of Technology, Tokyo 152-8550, Japan

(*correspondence: masuda.y.an@m.titech.ac.jp)

${ }^{2}$ Kwansei University, Hyogo 669-1337, Japan)

Micro-scale isotopic analysis of geologic materials is getting more important in recent geochemistry. Although insitu isotopic measurements with LA-ICP-MS or SIMS have played a central role in geochemical applications, these techniques are not necessarily suitable for isotopic analysis that requires chemical separation prior to mass spectrometry. In such cases, sampling with micro milling [1] or Laser Ablation in Liquid (LAL) [2] has been applied. However, these sampling techniques could cause cross contamination from the micro-drill material, relatively low recovery yield, and longer sampling time.

To overcome these problems, we developed the Laser Ablation with Filter (LAF) method in which sample particles ablated by a fs-laser (IFRIT, Cyber Laser) are carried via the flow of $\mathrm{He}$ gas in a Teflon tube and then caught by a membrane filter (pore size: $0.1 \mu \mathrm{m}$ ). The performance of the LAF method was evaluated by using a glass standard NIST 610. The glass was ablated by a raster speed of $100 \mu \mathrm{m} / \mathrm{s}$, which required $1.4 \mathrm{~h}$ for sampling an area of $1 \times 1 \times 0.1 \mathrm{~mm}^{3}$. The sample particles retrieved by the filter were dissolved by acid. The sample solution was split into two aliquots; one for the analysis of trace element abundances with ICP-MS (Xseries 2, Thermo) and the other for isotopic analysis with TIMS (TRITON plus, Thermo) after chemical separation. We found that the recovery yields of trace elements ranged from $80-90 \%$, in which the effect of elemental fluctuation was suppressed owing to the use of the fs-laser [2, 3]. The ${ }^{87} \mathrm{Sr} /{ }^{86} \mathrm{Sr}$ ratio of NIST 610 collected by the LAF method was $0.7096787 \pm 0.0000016$ (2SE), which is consistent with that for NIST $610\left({ }^{87} \mathrm{Sr} /{ }^{86} \mathrm{Sr}=0.7096779 \pm 0.0000028\right)$ separately measured by dissolving a piece of the glass standard. The procedural blank of the LAF method was negligible to perform the trace element and isotopic analyses. The new method can be applied to small minerals and inclusions in terrestrial rocks and meteorites.

[1] Myojo, K. et al. (2018) ApJ 853, 48. [2] Okabayashi, S. et al. (2011) JAAS, 26, 1393-1400. [3] Fernández B. et al. (2007) $\operatorname{Tr} A C 26,951-966$. 